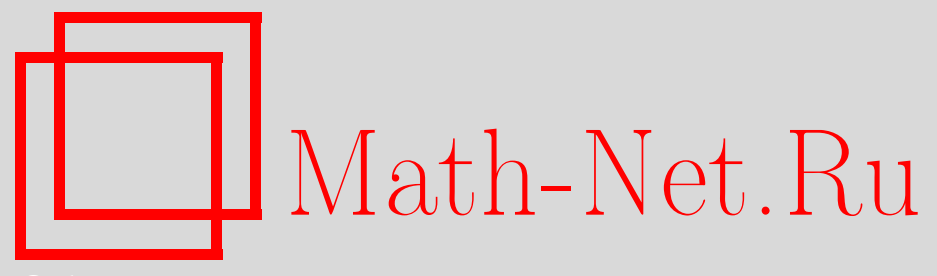

Е. В. Черепанова, Предельные распределения числа циклов заданной длины в случайной подстановке с известным числом циклов, Дискрет. матем., 2003, том 15, выпуск $3,128-144$

DOI: https://doi.org/10.4213/dm211

Использование Общероссийского математического портала Math-Net.Ru подразумевает, что вы прочитали и согласны с пользовательским соглашением http: //www.mathnet.ru/rus/agreement

Параметры загрузки:

IP : 54.197 .130 .99

26 апреля 2023 г., $12: 32: 20$

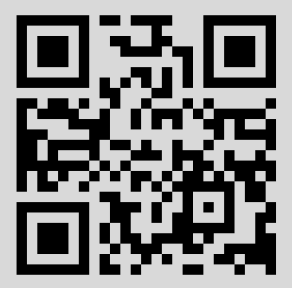




\title{
Предельные распределения числа циклов заданной длины в случайной подстановке с известным числом циклов
}

\author{
(c) 2003 г. $\quad$ Е. В. Черепанова
}

Получено полное описание предельного поведения числа циклов длины $r$ в случайной подстановке степени $n$, имеющей $N$ циклов, во всех зонах изменения параметров $n, N, r$. Доказанные в статье результаты существенно обобщают известные результаты о предельном поведении числа таких циклов.

Работа выполнена при поддержке гранта НШ 1758.2003.1 Президента Российской Федерации для государственной поддержки ведущих научных школ Российской Федерации.

Пусть $S_{n, N}$ - множество всех различных подстановок степени $n$, имеющих $N$ циклов. Введем на $S_{n, N}$ равномерное распределение вероятностей и обозначим через $\mu_{r}$ случайную величину, равную числу циклов длины $r$ в подстановке из $S_{n, N}$. В работе [1] получены предельные распределения $\mu_{r}$ при $n, N \rightarrow \infty$ в случаях, когда $r$ фиксировано и $n / N \rightarrow 1$ или $N / \ln n \rightarrow \gamma, 0<\gamma<\infty$, а также для всех $r$ при $1<c \leqslant n / N \leqslant c_{1}<\infty$, где $c, c_{1}$ - произвольные постоянные. В дальнейшем $c, C$, а также $c_{i}, C_{i}, i=1,2, \ldots$, будут обозначать положительные постоянные, не всегда различные.

В данной статье приводится полное описание предельного поведения $\mu_{r}$ во всех зонах изменения параметров $r, n, N$. Основным методом получения этих результатов является обобщенная схема размещения [2].

Введем независимые одинаково распределенные случайные величины $\xi_{1}, \ldots, \xi_{N}$ и $\xi_{1}^{(r)}, \ldots, \xi_{N}^{(r)}$ такие, что

$$
\begin{aligned}
p_{m}=\mathbf{P}\left\{\xi_{1}=m\right\} & =\frac{\lambda^{m}}{-m \ln (1-\lambda)}, \quad 0<\lambda<1, \quad m=1,2, \ldots, \\
\mathbf{P}\left\{\xi_{1}^{(r)}=m\right\} & =\mathbf{P}\left\{\xi_{1}=m \mid \xi_{1} \neq r\right\} .
\end{aligned}
$$

В [2] показано, что для любого $k=0,1, \ldots, N$

$$
\mathbf{P}\left\{\mu_{r}=k\right\}=\left(\begin{array}{l}
N \\
k
\end{array}\right) p_{r}^{k}\left(1-p_{r}\right)^{N-k} \frac{\mathbf{P}\left\{\zeta_{N-k}^{(r)}=n-k r\right\}}{\mathbf{P}\left\{\zeta_{N}=n\right\}},
$$


где

$$
\begin{aligned}
\zeta_{N} & =\xi_{1}+\ldots+\xi_{N}, \\
\zeta_{N-k}^{(r)} & =\xi_{1}^{(r)}+\ldots+\xi_{N-k}^{(r)} .
\end{aligned}
$$

Заметим, что равенство (3) справедливо при любом значении параметра $\lambda$ распределения (1), $0<\lambda<1$. В дальнейшем будем считать, что $\lambda=1-1 / n$ при $N / \ln n=O(1)$, а если $N / \ln n \rightarrow \infty$, то $\lambda$ - решение уравнения

$$
-\frac{\lambda}{(1-\lambda) \ln (1-\lambda)}=\frac{n}{N}
$$

в интервале $(0,1)$.

Справедливы следующие утверждения.

Теорема 1. Пусть $n, N \rightarrow \infty$ так, что выполнено одно из следующих условий:

(1) $n / N \geqslant c>1, N / \ln n \rightarrow \infty, N p_{r} \rightarrow \infty$;

(2) $n / N \rightarrow 1,(n-N)^{2} / N \rightarrow \infty, r=1$;

(3) $n / N \rightarrow 1,(n-N)^{3} / N^{2} \rightarrow \infty, r=2$;

(4) $n / N \rightarrow 1,(n-N)^{r-1} /\left(r N^{r-2}\right) \rightarrow \infty, r \geqslant 3$.

Tогдa

$$
\mathbf{P}\left\{\mu_{r}=k\right\}=\frac{1}{\sigma_{r r} \sqrt{2 \pi N}} e^{-u_{r}^{2} / 2}(1+o(1))
$$

равномерно относительно $k$, для которых

$$
u_{r}=\frac{k-N p_{r}}{\sigma_{r r} \sqrt{N}}
$$

лежит в любом фиксированном конечном интервале, где

$$
\begin{aligned}
\sigma_{r r}^{2} & =p_{r}\left(1-p_{r}-\frac{(n / N-r)^{2} p_{r}}{\sigma^{2}}\right), \\
\sigma^{2} & =\frac{n}{N}\left(\frac{1}{1-\lambda}-\frac{n}{N}\right) .
\end{aligned}
$$

Замечание 1. В случае, когда $1<c \leqslant n / N \leqslant c_{1}<\infty$ и $r$ фиксировано, утверждение теоремы 1 доказано в [1].

Теорема 2. Пусть выполнено одно из следующих условий:

(1) $n, N, r \rightarrow \infty$ так, что $n / N \geqslant c>1, N / \ln n \rightarrow \infty$;

(2) $n \rightarrow \infty, N=\gamma \ln n+o(\ln n), 0<\gamma<\infty$;

(3) $n, N \rightarrow \infty$ так, что $n / N \rightarrow 1, n-N \rightarrow \infty, r \geqslant 3$.

5 Дискретная математика, т.15 №3 
Тогда

$$
\mathbf{P}\left\{\mu_{r}=k\right\}=\frac{\left(N p_{r}\right)^{k}}{k !} e^{-N p_{r}}(1+o(1))
$$

равномерно относительно чельх неотрицательных $k$, для которых $\left(k-N p_{r}\right) / \sqrt{N p_{r}}$ лежит в любом фиксированном конечном интервале.

Замечание 2. Утверждение при условии (1) теоремы 2 и $n / N \leqslant c_{1}<\infty$, а также при условиях (2), (3) для фиксированных $r$, получено в [1].

Теорема 3. Пусть $n, N \rightarrow \infty$ так, что

$$
n / N \rightarrow 1, \quad \beta=4(n-N)^{2} / 3 N=O(1) .
$$

Тогда для любого фиксированного $k=0,1,2, \ldots$

$$
\begin{aligned}
& \mathbf{P}\left\{\mu_{1}=2 N-n+k\right\}=\frac{\beta^{k}}{k !} e^{-\beta}+o(1), \\
& \mathbf{P}\left\{\mu_{2}=n-N-2 k\right\}=\frac{\beta^{k}}{k !} e^{-\beta}+o(1) .
\end{aligned}
$$

Замечание 3. В случае фиксированной величины $n-N$ справедливость второго утверждения теоремы 3 доказана в [1].

Теорема 4. Пусть $n, N \rightarrow \infty$ так, что

$$
n / N \rightarrow 1, \quad \beta=4(n-N)^{2} /(3 N) \rightarrow \infty, \quad v=4(n-N)^{3} / N^{2}=O(1)
$$

Тогда

$$
\begin{array}{r}
\mathbf{P}\left\{\mu_{2}=n-N-2 k\right\}=\frac{\beta^{k}}{2 k !} e^{-\beta}\left(1+e^{-v}\right)+o\left(\frac{\beta^{k}}{k !} e^{-\beta}\right), \\
\mathbf{P}\left\{\mu_{2}=n-N-2 k-1\right\}=\frac{\beta^{k}}{2 k !} e^{-\beta}\left(1-e^{-v}\right)+o\left(\frac{\beta^{k}}{k !} e^{-\beta}\right)
\end{array}
$$

равномерно относительно цельх $k$, для которых $(k-\beta) / \sqrt{\beta}$ лежит в любом фиксированном конечном интервале.

Пусть $[x]$ означает целую часть числа $x$.

Теорема 5. Если

$$
N \geqslant 2, \quad n \rightarrow \infty, \quad N / \ln n \rightarrow 0, \quad r=[\alpha n], \quad 0<\alpha=\alpha(n) \leqslant c<1,
$$

$u 2 r \neq n$ nрu $N=2$, mo

$$
\begin{aligned}
& \mathbf{P}\left\{\mu_{r}=0\right\}=1-(N-1) p_{r} \frac{e^{\alpha}}{1-\alpha}+o\left(p_{r}\right), \\
& \mathbf{P}\left\{\mu_{r}=1\right\}=(N-1) p_{r} \frac{e^{\alpha}}{1-\alpha}+o\left(p_{r}\right) .
\end{aligned}
$$


Теорема 6. Пусть

$N \geqslant 3, \quad n \rightarrow \infty, \quad N / \ln n \rightarrow 0, \quad m=n-r \geqslant N-1, \quad m \rightarrow \infty, \quad m=o(n)$.

Тогда справедливы следующие утверждения:

(1) если $N=o(\ln n)$, mo

$$
\begin{aligned}
& \mathbf{P}\left\{\mu_{r}=0\right\}=1-\frac{N-1}{m \ln n}\left(\frac{\ln m}{\ln n}\right)^{N-2}(1+o(1)), \\
& \mathbf{P}\left\{\mu_{r}=1\right\}=\frac{N-1}{m \ln n}\left(\frac{\ln m}{\ln n}\right)^{N-2}(1+o(1)) ;
\end{aligned}
$$

(2) если $N / \ln m \rightarrow \gamma, 0<\gamma<\infty$, то

$$
\begin{aligned}
& \mathbf{P}\left\{\mu_{r}=0\right\}=1-\frac{1}{m \Gamma(\gamma)}\left(\frac{\ln m}{\ln n}\right)^{N-1}(1+o(1)), \\
& \mathbf{P}\left\{\mu_{r}=1\right\}=\frac{1}{m \Gamma(\gamma)}\left(\frac{\ln m}{\ln n}\right)^{N-1}(1+o(1)),
\end{aligned}
$$

где $\Gamma(\gamma)$ - значение гамма-функции в точке $\gamma$;

(3) если $N / \ln m \rightarrow \infty, \alpha=m / N \rightarrow \infty$, то

$$
\begin{aligned}
& \mathbf{P}\left\{\mu_{r}=0\right\}=1-\frac{1}{x_{\alpha}^{m} \sigma_{\alpha} \sqrt{2 \pi N}}\left(-\frac{\ln \left(1-x_{\alpha}\right)}{\ln n}\right)^{N-1}(1+o(1)), \\
& \mathbf{P}\left\{\mu_{r}=1\right\}=\frac{1}{x_{\alpha}^{m} \sigma_{\alpha} \sqrt{2 \pi N}}\left(-\frac{\ln \left(1-x_{\alpha}\right)}{\ln n}\right)^{N-1}(1+o(1)),
\end{aligned}
$$

где

$$
x_{\alpha}=1-(\alpha \ln \alpha)^{-1}, \quad \sigma_{\alpha}=\alpha \sqrt{\ln \alpha}
$$

(4) если $N / \ln m \rightarrow \infty, 1<c_{1} \leqslant \alpha=m / N \leqslant c_{2}<\infty$, то

$$
\begin{aligned}
& \mathbf{P}\left\{\mu_{r}=0\right\}=1-\frac{1}{x^{m} \sigma(x) \sqrt{2 \pi N}}\left(-\frac{\ln (1-x)}{\ln n}\right)^{N-1}(1+o(1)), \\
& \mathbf{P}\left\{\mu_{r}=1\right\}=\frac{1}{x^{m} \sigma(x) \sqrt{2 \pi N}}\left(-\frac{\ln (1-x)}{\ln n}\right)^{N-1}(1+o(1)),
\end{aligned}
$$

где $x$-- решение уравнения

$$
-\frac{x}{(1-x) \ln (1-x)}=\frac{m}{N-1}
$$

в интервале $(0,1)$,

$$
\sigma^{2}(x)=-\frac{x \ln (1-x)+x^{2}}{(1-x)^{2} \ln ^{2}(1-x)}
$$


(5) если $N / \ln m \rightarrow \infty, \alpha=m / N \rightarrow 1, m-N+1 \geqslant c_{3} m^{c_{4}}, c_{3}>0,0<c_{4}<1$, mo

$$
\begin{aligned}
& \mathbf{P}\left\{\mu_{r}=0\right\}=1-\frac{1}{x^{m} \sqrt{2 \pi(m-N)}}\left(-\frac{\ln (1-x)}{\ln n}\right)^{N-1}(1+o(1)), \\
& \mathbf{P}\left\{\mu_{r}=1\right\}=\frac{1}{x^{m} \sqrt{2 \pi(m-N)}}\left(-\frac{\ln (1-x)}{\ln n}\right)^{N-1}(1+o(1)),
\end{aligned}
$$

где $x$-решение уравнения (5) в интервале $(0,1)$;

(6) если $m-\delta \sqrt{m} \leqslant N-1 \leqslant m, \delta \rightarrow 0$, mo

$$
\begin{aligned}
& \mathbf{P}\left\{\mu_{r}=0\right\}=1-\frac{1}{(m-N+1) !(\ln n)^{N-1}}\left(\frac{N-1}{2}\right)^{m-N+1}(1+o(1)), \\
& \mathbf{P}\left\{\mu_{r}=1\right\}=\frac{1}{(m-N+1) !(\ln n)^{N-1}}\left(\frac{N-1}{2}\right)^{m-N+1}(1+o(1)) .
\end{aligned}
$$

Замечание 4. Утверждение 1 теоремы 6 остается справедливым, если $N=2, m=o(n)$, причем $m$ может быть фиксированным.

Теорема 7. Пусть

$$
N \geqslant 3, \quad m=n-r \geqslant N-1,
$$

$N$ и ти фиксированы, $n \rightarrow \infty$. Тогда

$$
\begin{aligned}
& \mathbf{P}\left\{\mu_{r}=0\right\}=1-\frac{1}{(\ln n)^{N-1}} \sum \frac{1}{k_{1} \ldots k_{N-1}}+o\left(\frac{1}{(\ln n)^{N-1}}\right), \\
& \mathbf{P}\left\{\mu_{r}=1\right\}=\frac{1}{(\ln n)^{N-1}} \sum \frac{1}{k_{1} \ldots k_{N-1}}+o\left(\frac{1}{(\ln n)^{N-1}}\right),
\end{aligned}
$$

где суммирование проводится по всем натуральным $k_{1}, k_{2}, \ldots, k_{N-1}$ таким, что $k_{1}+\ldots+k_{N-1}=m$.

Теорема 8. Если

$$
N=2, \quad n \rightarrow \infty, \quad 2 r=n,
$$

mo

$$
\begin{aligned}
& \mathbf{P}\left\{\mu_{r}=0\right\}=1-p_{r} \sqrt{e}+o\left(p_{r}\right), \\
& \mathbf{P}\left\{\mu_{r}=2\right\}=p_{r} \sqrt{e}+o\left(p_{r}\right) .
\end{aligned}
$$

Введем обозначения

$$
\begin{aligned}
m_{r} & =\mathbf{M} \xi_{1}^{(r)} \\
\sigma_{r}^{2} & =\mathbf{D} \xi_{1}^{(r)} \\
\varphi(t) & =\mathbf{M} \exp \left\{i t \xi_{1}\right\}, \\
\varphi_{r}(t) & =\mathbf{M} \exp \left\{i t \xi_{1}^{(r)}\right\}, \\
\psi_{s}^{(r)}(t) & =\mathbf{M} \exp \left\{i t\left(\frac{\zeta_{s}^{(r)}-s m_{r}}{\sigma_{r} \sqrt{s}}\right)\right\} .
\end{aligned}
$$


Нетрудно видеть, что

$$
\begin{aligned}
m_{r} & =\frac{1}{1-p_{r}}\left(-\frac{\lambda}{(1-\lambda) \ln (1-\lambda)}-r p_{r}\right), \\
\sigma_{r}^{2} & =\frac{1}{1-p_{r}}\left(-\frac{\lambda}{(1-\lambda)^{2} \ln (1-\lambda)}-r^{2} p_{r}\right)-m_{r}^{2}, \\
\varphi(t) & =\frac{\ln \left(1-\lambda e^{i t}\right)}{\ln (1-\lambda)} \\
\varphi_{r}(t) & =\frac{1}{1-p_{r}}\left(\varphi(t)-p_{r} e^{i t r}\right), \\
\psi_{s}^{(r)}(t) & =\exp \left\{-\frac{i t s m_{r}}{\sigma_{r} \sqrt{s}}\right\} \varphi_{r}^{s}\left(\frac{t}{\sigma_{r} \sqrt{s}}\right) .
\end{aligned}
$$

Ниже приводятся вспомогательные утверждения (леммы 1-4), а затем с их помощью будут доказаны теоремы 1-8.

Лемма 1. Пусть $n, N \rightarrow \infty$,

$$
s=N\left(1-p_{r}\right)(1+o(1))
$$

и выполнено одно из следующих условий:

(1) $n / N \geqslant c>1, N / \ln n \rightarrow \infty$;

(2) $n / N \rightarrow 1,(n-N)^{2} / N \rightarrow \infty, r=1,2$;

(3) $n / N \rightarrow 1, n-N \rightarrow \infty, r \geqslant 3$.

Тогда последовательность распределений сумм $\left(\zeta_{s}^{(r)}-s m_{r}\right) / \sigma_{r} \sqrt{s}$ слабо сходится $\kappa$ стандартному нормальному закону.

Доказательство. Если выполнено условие (1) леммы и, кроме того, $n / N \leqslant C_{1}<\infty$, то решение уравнения (4) находится в интервале вида $0<C_{2} \leqslant \lambda \leqslant C_{3}<1$, и для любого $r$ справедлива оценка

$$
\sigma_{r}^{2} \geqslant C_{4}>0
$$

а если $n / N \rightarrow \infty$, то, как отмечено в [3], уравнение (4) приближенно удовлетворяется при

$$
\lambda=1-\left(\frac{n}{N} \ln \frac{n}{N}\right)^{-1},
$$

и из (6), (7) получаем, что при таком выборе $\lambda$ для всех $r$

$$
\sigma_{r}^{2}=\left(\frac{n}{N}\right)^{2} \ln \frac{n}{N}(1+o(1)) .
$$

При достаточно малых $t$ имеет место равенство

$$
\varphi_{r}(t)=1+i m_{r} t-\frac{\sigma_{r}^{2}+m_{r}^{2}}{2} t^{2}+\frac{\varphi_{r}^{\prime \prime \prime}(\delta)}{6} t^{3},
$$


где $0<\delta<|t|$ и

$$
\left|\varphi_{r}^{\prime \prime \prime}(\delta)\right| \leqslant \mathbf{M}\left(\xi_{1}^{(r)}\right)^{3}=\frac{1}{1-p_{r}}\left(\frac{1+\lambda}{(1-\lambda)^{2}} \frac{n}{N}-r^{3} p_{r}\right) .
$$

Отсюда и из (10), (11), (13) следует, как легко проверить, что при любом фиксированном $t$ справедливо соотношение

$$
\psi_{s}^{(r)}(t) \rightarrow e^{-t^{2} / 2}
$$

Таким образом, при выполнении условия (1) лемма доказана.

Пусть $n / N \rightarrow 1$. Из (4) получаем, что

$$
\lambda=2\left(\frac{n-N}{N}\right)-\frac{10}{3}\left(\frac{n-N}{N}\right)^{2}+\frac{16}{9}\left(\frac{n-N}{N}\right)^{3}+o\left(\left(\frac{n-N}{N}\right)^{3}\right) .
$$

Учитывая (1), (6), (7), (16), несложно показать, что

$$
\begin{array}{rlrl}
1-p_{1} & =\left(\frac{n-N}{N}\right)(1+o(1)), \quad 1-p_{r} \rightarrow 1, \quad & r \geqslant 2, \\
\sigma_{1}^{2} & =\frac{4}{3}\left(\frac{n-N}{N}\right)(1+o(1)), & & \\
\sigma_{2}^{2} & =\frac{16}{3}\left(\frac{n-N}{N}\right)^{2}(1+o(1)), & & \\
\sigma_{r}^{2} & =\left(\frac{n-N}{N}\right)(1+o(1)), & & r \geqslant 3 .
\end{array}
$$

Используя (8), (9), можно найти, что при выполнении условий леммы (2), (3) и при $t \rightarrow 0$

$$
\begin{aligned}
\varphi_{1}(t)= & e^{2 i t}\left(1+\frac{i t}{1-p_{1}}\left(\frac{n}{N} \lambda+2 p_{1}-2\right)\right. \\
& \left.-\frac{t^{2}}{2\left(1-p_{1}\right)}\left(\frac{n}{N(1-\lambda)}-(3 \lambda+1) \frac{n}{N}-4 p_{1}+4\right)+O\left(\lambda t^{3}\right)\right), \\
\varphi_{r}(t)= & e^{i t}\left(1+\frac{i t}{1-p_{r}}\left(\frac{n}{N}-1-(r-1) p_{r}\right)\right. \\
& \left.-\frac{t^{2}}{2\left(1-p_{r}\right)}\left(\frac{n}{N(1-\lambda)}-2 \frac{n}{N}+1-(r-1)^{2} p_{r}\right)+O\left(t^{3} \lambda^{2+\operatorname{sign}(2-r)}\right)\right), r \geqslant 2 .
\end{aligned}
$$

Из (6), (7), (10), (16)-(18) следует, что при всех $r \geqslant 1$ и любом фиксированном $t$ справедливо соотношение (15), что и завершает доказательство леммы.

Лемма 2. Пусть выполнены условия леммы 1 и, кроме того, $(n-N)^{3} / N^{2} \rightarrow \infty$ при $r=2$. Тогда

$$
\mathbf{P}\left\{\zeta_{s}^{(r)}=l\right\}=\frac{1}{\sigma_{r} \sqrt{2 \pi s}} e^{-z^{2} / 2}(1+o(1))
$$

равномерно по $l$, для которых $z=\left(l-s m_{r}\right) /\left(\sigma_{r} \sqrt{s}\right)$ лежит в любом фиксированном конечном интервале. 
Доказательство. По формуле обрашения, вероятность $\mathbf{P}\left\{\zeta_{s}^{(r)}=l\right\}$ можно представить в виде

$$
\mathbf{P}\left\{\zeta_{s}^{(r)}=l\right\}=\frac{1}{2 \pi \sigma_{r} \sqrt{s}} \int_{-\pi \sigma_{r} \sqrt{s}}^{\pi \sigma_{r} \sqrt{s}} e^{-i z t} \psi_{s}^{(r)}(t) d t .
$$

Поскольку

$$
\frac{1}{\sqrt{2 \pi}} e^{-z^{2} / 2}=\frac{1}{2 \pi} \int_{-\infty}^{\infty} e^{-i z t} e^{-t^{2} / 2} d t
$$

получаем, что

$$
R=\sqrt{2 \pi}\left(\sigma_{r} \sqrt{2 \pi s} \mathbf{P}\left\{\zeta_{s}^{(r)}=l\right\}-e^{-z^{2} / 2}\right)=I_{1}+I_{2}+I_{3}+I_{4},
$$

где

$$
\begin{aligned}
& I_{1}=\int_{|t| \leqslant A} e^{-i z t}\left(\psi_{s}^{(r)}(t)-e^{-t^{2} / 2}\right) d t, \\
& I_{2}=\int_{A<|t| \leqslant \varepsilon \sigma_{r} \sqrt{s}} e^{-i z t} \psi_{s}^{(r)}(t) d t, \\
& I_{3}=\int_{\varepsilon \sigma_{r} \sqrt{s}<|t| \leqslant \pi \sigma_{r} \sqrt{s}} e^{-i z t} \psi_{s}^{(r)}(t) d t, \\
& I_{4}=-\int_{|t| \geqslant A} e^{-i z t-t^{2} / 2} d t,
\end{aligned}
$$

положительные постоянные $A, \varepsilon$ будут выбраны позднее. Для доказательства леммы достаточно показать, что разность $R$ можно сделать сколь угодно малой при больших $n, N$.

В силу леммы 1 равномерно в любом конечном интервале справедливо соотношение (15), следовательно, $I_{1} \rightarrow 0$ для каждого фиксированного $A>0$.

Поскольку

$$
\left|I_{4}\right| \leqslant \int_{|t| \geqslant A} e^{-t^{2} / 2} d t,
$$

выбором достаточно большого $A$ интеграл $I_{4}$ можно сделать сколь угодно малым.

Оценим $I_{2}$. Если

$$
1<c \leqslant n / N \leqslant C_{1}<\infty
$$

то для $|t| \leqslant \varepsilon$ при достаточно малом $\varepsilon$ из (10) и (14) получаем, что

$$
\left|\psi_{s}^{(r)}(t)\right| \leqslant e^{-t^{2} / 4}
$$

для любого $r$. Тогда

$$
\left|I_{2}\right| \leqslant \int_{A<|t| \leqslant \varepsilon \sigma_{r} \sqrt{s}} e^{-t^{2} / 4} d t \leqslant 2 \int_{A}^{\infty} e^{-t^{2} / 4} d t
$$

и интеграл $I_{2}$ можно сделать сколь угодно малым, выбрав $A$ достаточно большим.

Пусть $n / N \rightarrow \infty, N / \ln n \rightarrow \infty$. Представим $I_{2}$ в виде суммы

$$
I_{2}=I_{2}^{\prime}+I_{2}^{\prime \prime}+I_{2}^{\prime \prime \prime}
$$


где областями интегрирования интегралов $I_{2}^{\prime}, I_{2}^{\prime \prime}, I_{2}^{\prime \prime \prime}$ соответственно являются

$$
\begin{aligned}
& S_{1}=\{A<|t| \leqslant \varepsilon \sqrt{N / \ln n}\}, \\
& S_{2}=\left\{\varepsilon \sqrt{N / \ln n}<|t| \leqslant \min \left\{N / \ln n, \varepsilon \sigma_{r} \sqrt{s}\right\},\right. \\
& S_{3}=\left\{\min \left\{N / \ln n, \varepsilon \sigma_{r} \sqrt{s}\right\}<|t| \leqslant \varepsilon \sigma_{r} \sqrt{s}\right\},
\end{aligned}
$$

при этом, если $N / \ln n \geqslant \varepsilon \sigma_{r} \sqrt{s}$, то область $S_{3}$ пуста. Для $t \in S_{1}$ при достаточно малом $\varepsilon$ из (10) и (14) получаем, что для всех $r$ имеет место оценка (19) и, по аналогии с (20), интеграл $I_{2}^{\prime}$ можно сделать сколь угодно малым.

Функцию $\psi_{s}^{(r)}(t)$ можно представить в виде

$$
\psi_{s}^{(r)}(t)=\exp \left\{-\frac{i t s m_{r}}{\sigma_{r} \sqrt{s}}\right\} \varphi^{s}\left(\frac{t}{\sigma_{r} \sqrt{s}}\right)\left(1+\frac{p_{r}}{1-p_{r}}\left(1-\frac{e^{i t r / \sigma_{r} \sqrt{s}}}{\varphi\left(t / \sigma_{r} \sqrt{s}\right)}\right)\right)^{s} .
$$

Нетрудно видеть, что если $|t|<\varepsilon$ и $\varepsilon$ достаточно мало, то для любого $r$ справедливы соотношения

$$
\begin{aligned}
1+\frac{p_{r}}{1-p_{r}}\left(1-\frac{e^{i t r}}{\varphi(t)}\right) & =1+o\left(\frac{1}{\ln (1-\lambda)}\right) \\
\frac{\ln \left|1-\lambda e^{i t}\right|}{\ln (1-\lambda)} & =1+\frac{\ln \left(1+\lambda(t /(1-\lambda))^{2}+o\left((1 /(1-\lambda))^{2}\right)\right)}{2 \ln (1-\lambda)} .
\end{aligned}
$$

Если $t \in S_{2}$, то из (12), (13) и (23) находим, что

$$
\begin{gathered}
\frac{|t|}{(1-\lambda) \sigma_{r} \sqrt{s}} \geqslant C_{2}, \\
\frac{\ln \mid 1-\lambda e^{i t / \sigma_{r} \sqrt{s} \mid}}{\ln (1-\lambda)} \leqslant 1+\frac{C_{3}}{\ln (1-\lambda)} .
\end{gathered}
$$

Из последнего неравенства и (12), (21), (22) несложно получить, что для всех $r$

$$
\left|\psi_{s}^{(r)}(t)\right| \leqslant \exp \left\{-C_{4} N / \ln n\right\}
$$

Тогда

$$
\left|I_{2}^{\prime \prime}\right| \leqslant C_{5} \frac{N}{\ln n} \exp \left\{-C_{4} N / \ln n\right\}
$$

и, очевидно, $I_{2}^{\prime \prime} \rightarrow 0$ при $N / \ln n \rightarrow \infty$.

Если $t \in S_{3}$, то, учитывая (12), (13), нетрудно видеть, что

$$
|t| /(1-\lambda) \sigma_{r} \sqrt{s} \geqslant C_{6} \sqrt{N / \ln n} \rightarrow \infty,
$$

и из (8), (23) следует, что

$$
\varphi\left(\frac{t}{\sigma_{r} \sqrt{s}}\right)=1+\frac{\ln \left(|t| /(1-\lambda) \sigma_{r} \sqrt{s}\right)}{\ln (1-\lambda)}+i \frac{\arg \left(1-\lambda e^{i t / \sigma_{r} \sqrt{s}}\right)}{\ln (1-\lambda)}+o\left(\frac{1}{\ln (1-\lambda)}\right) .
$$


Отсюда и из (21), (22) находим, что

$$
\begin{aligned}
\left|\psi_{s}^{(r)}(t)\right| & \leqslant\left(1+C_{7} \frac{\ln \left(|t| /(1-\lambda) \sigma_{r} \sqrt{s}\right)}{\ln (1-\lambda)}\right)^{s / 2} \\
& \leqslant\left(\frac{|t|}{(1-\lambda) \sigma_{r} \sqrt{s}}\right)^{C_{8} s / \ln (1-\lambda)}
\end{aligned}
$$

Тогда

$$
\begin{aligned}
\left|I_{2}^{\prime \prime \prime}\right| & \leqslant 2 \int_{N / \ln n}^{\varepsilon \sigma_{r} \sqrt{s}}\left(\frac{t}{(1-\lambda) \sigma_{r} \sqrt{s}}\right)^{C_{8 s} / \ln (1-\lambda)} d t \\
& =2(1-\lambda) \sigma_{r} \sqrt{s} \int_{N /(1-\lambda) \sigma_{r} \sqrt{s} \ln n}^{\mathcal{E} /(1-\lambda)} y^{C_{8 . s} / \ln (1-\lambda)} d y,
\end{aligned}
$$

и, используя (12), (13) и соотношение $N / \ln n \rightarrow \infty$, получаем, что $I_{2}^{\prime \prime \prime} \rightarrow 0$.

Таким образом, если выполнено условие (1) леммы 1 , то интеграл $I_{2}$ можно сделать сколь угодно малым при достаточно больших $n, N, A$ и малом $\varepsilon$.

При выполнении условий (2), (3) леммы 1 из соотношений (10), (16)-(18) получаем, что для всех $r \geqslant 1$ в области интегрирования $I_{2}$ при достаточно малом $\varepsilon$ справедливо неравенство (19). Следовательно, верно (20), и интеграл $I_{2}$ можно сделать сколь угодно малым выбором достаточно большого $A$.

Остается оценить $I_{3}$. Пусть $1<c \leqslant n / N \leqslant C_{1}<\infty$. Поскольку максимальный шаг распределения $\xi_{1}^{(r)}$ равен 1 и $\varphi_{r}(t)$ непрерывно зависит от параметра $\lambda$, $0<C_{9} \leqslant \lambda \leqslant C_{10}<1$, при фиксированных $r$ и $\varepsilon<|t| \leqslant \pi$ выполняется неравенство

$$
\left|\varphi_{r}(t)\right| \leqslant e^{-C_{11}} .
$$

Аналогично, для характеристической функции $\varphi(t)$ случайной величины $\xi_{1}$ справедлива оценка вида (24). Если $r \rightarrow \infty$, то $p_{r} \rightarrow 0$ и, используя (9) и оценку для $\varphi(t)$, находим, что при $\varepsilon<|t| \leqslant \pi$

$$
\left|\varphi_{r}(t)\right| \leqslant|\varphi(t)|\left|1+\frac{p_{r}}{1-p_{r}}\left(1-\frac{e^{i t r}}{\varphi(t)}\right)\right| \leqslant e^{-C_{11}} .
$$

Поскольку $\sigma_{r} \leqslant C_{12}$, получаем, что

$$
\begin{aligned}
\left|I_{3}\right| & \leqslant \int_{\varepsilon \sigma_{r} \sqrt{s}<|t| \leqslant \pi \sigma_{r} \sqrt{s}}\left|\varphi_{r}\left(\frac{t}{\sigma_{r} \sqrt{s}}\right)\right|^{s} d t \\
& \leqslant 2 \int_{\varepsilon \sigma_{r} \sqrt{s}}^{\pi \sigma_{r} \sqrt{s}} e^{-C_{11 s} s} d t \leqslant C_{13} \sigma_{r} \sqrt{s} e^{-C_{11} s} \rightarrow 0 .
\end{aligned}
$$

Пусть $n / N \rightarrow \infty$. Функщию $\varphi(t)$ представим в виде (см. [3])

$$
\varphi(t)=\frac{\ln (1-\lambda-i t)+\ln \left(1+f_{1}(t)+f_{2}(t)\right)}{\ln (1-\lambda)},
$$

где

$$
\begin{array}{ll}
f_{1}(t)=\frac{1+i t-e^{i t}}{1-\lambda-i t}, & \left|f_{1}(t)\right| \leqslant|t| / \sqrt{2}, \\
f_{2}(t)=\frac{(1-\lambda)\left(e^{i t}-1\right)}{1-\lambda-i t}, & \left|f_{2}(t)\right| \leqslant \sqrt{2}(1-\lambda) .
\end{array}
$$


Используя эти соотношения, а также (1), (9), (12), несложно показать, что при $\varepsilon<|t| \leqslant \pi$ справедливо неравенство (24). Отсюда и из (10), (13) находим, что

$$
\begin{aligned}
\left|I_{3}\right| & \leqslant \int_{\varepsilon \sigma_{r} \sqrt{s}<|t| \leqslant \pi \sigma_{r} \sqrt{s}} e^{-C_{11} s} d t \leqslant C_{14} \sigma_{r} \sqrt{s} e^{-C_{11} s} \\
& \leqslant C_{15} \sqrt{\frac{\ln n}{N}} \exp \left\{-C_{16} N\left(1-\frac{\ln n}{C_{16} N}\right)\right\}
\end{aligned}
$$

и $I_{3} \rightarrow 0$, если $N / \ln n \rightarrow \infty$.

При выполнении условий (2), (3) леммы $1, r \neq 2$ и $\varepsilon \sigma_{r} \sqrt{s}<|t| \leqslant \pi \sigma_{r} \sqrt{s}$ из (8), (9) получаем, что

$$
\begin{aligned}
& \left|\varphi_{1}\left(\frac{t}{\sigma_{1} \sqrt{s}}\right)\right|^{s}=\left|1+\frac{2}{3} \lambda\left(e^{i t / \sigma_{1} \sqrt{s}}-1\right)+o(\lambda)\right|^{s} \leqslant e^{-C_{17} \lambda s}, \\
& \left|\varphi_{r}\left(\frac{t}{\sigma_{r} \sqrt{s}}\right)\right|^{s}=\left|1+\frac{\lambda}{2}\left(e^{i t / \sigma_{r} \sqrt{s}}-1\right)+o(\lambda)\right|^{s} \leqslant e^{-C_{17} \lambda s}, \quad r \geqslant 3 .
\end{aligned}
$$

Отсюда и из (16), (17) следует, что $I_{3} \rightarrow 0$ при $n, N \rightarrow \infty$. Если $r=2$, то из (8), (9) находим, что при $\varepsilon<|t| \leqslant \pi$

$$
\begin{aligned}
\left|\varphi_{2}(t)\right|^{2} & =\left|1+\frac{\lambda^{2}}{3} e^{2 i t}+\frac{\lambda^{3}}{4} e^{3 i t}+O\left(\lambda^{4}\right)\right|\left(1-\frac{2}{3} \lambda^{2}-\frac{\lambda^{3}}{2}+O\left(\lambda^{4}\right)\right) \\
& =1-\frac{2}{3} \lambda^{2}(1-\cos 2 t)-\frac{\lambda^{3}}{2}(1-\cos 3 t)+O\left(\lambda^{4}\right) .
\end{aligned}
$$

Интеграл $I_{3}$ представим в виде суммы $I_{3}=I_{3}^{\prime}+I_{3}^{\prime \prime}$, где областями интегрирования интегралов $I_{3}^{\prime}, I_{3}^{\prime \prime}$ соответственно являются

$$
\left\{\varepsilon \sigma_{2} \sqrt{s}<|t| \leqslant(\pi-\varepsilon) \sigma_{2} \sqrt{s}\right\}, \quad\left\{(\pi-\varepsilon) \sigma_{2} \sqrt{s}<|t| \leqslant \pi \sigma_{2} \sqrt{s}\right\} .
$$

Из (25) следует, что если $\varepsilon<|t| \leqslant \pi-\varepsilon$, то

$$
\left|\varphi_{2}(t)\right| \leqslant e^{-C_{18} \lambda^{2}},
$$

а при $\pi-\varepsilon<|t| \leqslant \pi$

$$
\begin{aligned}
\left|\varphi_{2}(t)\right| & \leqslant\left(1-\frac{2}{3} \lambda^{2}(1-\cos 2 t)-2 C_{19} \lambda^{3}\right)^{1 / 2} \\
& \leqslant \exp \left\{-C_{20} \lambda^{2}(t-\pi)^{2}-C_{19} \lambda^{3}\right\} .
\end{aligned}
$$

Используя эти неравенства и (10), несложно получить, что

$$
\begin{aligned}
\left|I_{3}^{\prime}\right| & \leqslant C_{21} \sigma_{2} \sqrt{s} e^{-C_{18} \lambda^{2} s} \\
\left|I_{3}^{\prime \prime}\right| & \leqslant \sigma_{2} \sqrt{s} e^{-C_{19} \lambda^{3} s} \int_{-\varepsilon}^{\varepsilon} e^{-C_{20} \lambda^{2} s t^{2}} d t \\
& =\frac{\sigma_{2}}{\lambda} e^{-C_{19} \lambda^{3} s} \int_{-\varepsilon \lambda \sqrt{s}}^{\varepsilon \lambda \sqrt{s}} e^{-C_{20} u^{2}} d u .
\end{aligned}
$$

Отсюда, из (16), (17) и соотношения $(n-N)^{3} / N^{2} \rightarrow \infty$ следует, что $I_{3} \rightarrow 0$ при $n, N \rightarrow \infty$. Лемма доказана. 
Повторяя доказательства теорем 4.2.1 и 4.2.3 из книги [3], можно показать, что справедливы следующие утверждения.

Лемма 3. Пусть

$$
n \rightarrow \infty, \quad N=\gamma \ln n+o(\ln n), \quad 0<\gamma<\infty, \quad s=N\left(1-p_{r}\right)(1+o(1)) .
$$

Тогда

$$
\mathbf{P}\left\{\zeta_{s}^{(r)}=l\right\}=\frac{1}{n \Gamma(\gamma)} z^{\gamma-1} e^{-z}(1+o(1))
$$

равномерно относительно $l$, для которых $z=l / n$ лежит в любом фиксированном интервале вида $0<z_{1} \leqslant z \leqslant z_{2}$, где $z_{1}, z_{2}$ - постоянные.

Лемма 4. Пусть

$$
n \rightarrow \infty, \quad N / \ln n \rightarrow 0, \quad l=[\beta n], \quad 0<\varepsilon \leqslant \beta \leqslant 1 .
$$

Тогда

$$
\mathbf{P}\left\{\zeta_{N}=l\right\}=N p_{l}(1+o(1))=\frac{N e^{-\beta}}{\beta n \ln n}(1+o(1)) .
$$

Теперь докажем теоремы 1-8.

Пусть выполнены условия теоремы 1 или 2. Если $\left(k-N p_{r}\right) / \sqrt{N p_{r}\left(1-p_{r}\right)}$ лежит в конечном фиксированном интервале, то, как легко видеть,

$$
s=N-k=N\left(1-p_{r}\right)(1+o(1))
$$

при $N p_{r}\left(1-p_{r}\right) \rightarrow \infty$ или $p_{r} \rightarrow 0$. Следовательно, в условиях теорем 1,2 справедливы леммы 1-3. Используя для оценки $\mathbf{P}\left\{\zeta_{N}=n\right\}$ теоремы 4.2.1, 4.2.5, 4.2.7, 4.2 .9 из [3], утверждение теоремы 1 получаем из (3), леммы 2, соотношения $N p_{r}\left(1-p_{r}\right) \rightarrow \infty$ и нормального приближения биномиальных вероятностей, а утверждение теоремы 2 из (3), лемм 2, 3, соотношения $p_{r} \rightarrow 0$ и пуассоновского приближения биномиальных вероятностей.

Доказательство теоремы 3. В статье [4] изучалось предельное поведение максимального объема дерева в случайном рекурсивном лесе, состоящем из $N$ деревьев и $n$ вершин, при $n, N \rightarrow \infty$, было показано, что распределения объемов деревьев в таком лесе совпадают с распределениями длин циклов в подстановке из $S_{n, N}$. Из теоремы 1 этой статьи следует, что

$$
\begin{aligned}
& \mathbf{P}\left\{\eta_{(N)}=2\right\}=e^{-\beta}+o(1), \\
& \mathbf{P}\left\{\eta_{(N)}=3\right\}=1-e^{-\beta}+o(1),
\end{aligned}
$$

где $\eta_{(N)}$ - максимальная длина цикла в подстановке из $S_{n, N}$. Учитывая эти равенства, получаем, что

$$
\mathbf{P}\left\{\mu_{1}=m_{1}\right\}=\sum \mathbf{P}\left\{\mu_{1}=m_{1}, \mu_{2}=m_{2}, \mu_{3}=m_{3}, \mu_{i}=0, i \geqslant 4\right\}+o(1),
$$


где суммирование проводится по всем целым неотрицательным $m_{2}, m_{3}$ таким, что

$$
m_{2}+m_{3}=N-m_{1}, \quad 2 m_{2}+3 m_{3}=n-m_{1} .
$$

Положив

$$
m_{1}=2 N-n+k, \quad k=0,1, \ldots,
$$

из (26) находим, что

$$
m_{2}=n-N-2 k, \quad m_{3}=k
$$

Следовательно,

$$
\begin{aligned}
& \mathbf{P}\left\{\mu_{1}=2 N-n+k\right\}=\mathbf{P}\left\{\mu_{3}=k\right\}+o(1) \\
& \mathbf{P}\left\{\mu_{2}=n-N-2 k\right\}=\mathbf{P}\left\{\mu_{3}=k\right\}+o(1) .
\end{aligned}
$$

Отсюда, из теоремы 2 и соотношения

$$
N p_{3}=\beta(1+o(1))
$$

получаем утверждение теоремы 3.

Доказательство теоремь 4. Легко видеть, что

$$
\mathbf{P}\left\{\mu_{2}=m_{2}\right\}=\sum \mathbf{P}\left\{\mu_{1}=m_{1}, \mu_{2}=m_{2}, \mu_{3}=m_{3}, \mu_{4}=m_{4}, \mu_{i}=0, i \geqslant 5\right\}+R,
$$

где

$$
R \leqslant \mathbf{P}\left\{\sum_{i \geqslant 5} i \mu_{i} \geqslant 5\right\},
$$

а суммирование проводится по всем целым неотрицательным $m_{1}, m_{3}, m_{4}$ таким, что

$$
m_{1}+m_{3}+m_{4}=N-m_{2}, \quad m_{1}+3 m_{3}+4 m_{4}=n-2 m_{2} .
$$

Используя теорему 2 и неравенство Чебышева, получаем оценку

$$
R \leqslant C_{1}(n-N)^{4} / N^{3}
$$

Отсюда, как несложно проверить, следует, что при выполнении условий теоремы

$$
R=o\left(\frac{\beta^{k}}{k !} e^{-\beta}\right) \text {. }
$$

Поскольку (см., например, [1]) число подстановок в $S_{n, N}$ равно $|s(n, N)|$, где $s(n, N)-$ числа Стирлинга первого рода, нетрудно видеть, что

$$
\begin{aligned}
\mathbf{P}\left\{\mu_{1}=m_{1}, \mu_{2}=m_{2}, \mu_{3}=m_{3}, \mu_{4}=m_{4}, \mu_{i}\right. & =0, i \geqslant 5\} \\
& =\frac{n !}{m_{1} ! m_{2} ! m_{3} ! m_{4} ! 2^{m_{2} 3^{m_{3}} 4^{m_{4}}|s(n, N)|}} .
\end{aligned}
$$


Положив

$$
m_{2}=n-N-2 k, \quad m_{4}=2 m, \quad m \geqslant 0,
$$

из (28) находим, что

$$
m_{1}=2 N-n+k+m, \quad m_{3}=k-3 m, \quad m \leqslant k / 3 .
$$

Тогда из (27), (29), (30) следует, что

$$
\mathbf{P}\left\{\mu_{2}=n-N-2 k\right\}=Z S+o\left(\frac{\beta^{k}}{k !} e^{-\beta}\right),
$$

где

$$
\begin{aligned}
& Z=\left(\frac{4}{3}\right)^{k} \frac{n !}{k !(2 N-n+k) !(n-N-2 k) ! 2^{n-N}|s(n, N)|} \\
& S=\sum_{m=0}^{[k / 3\rfloor}\left(\frac{27}{16}\right)^{m} \frac{k !(2 N-n+k) !}{(2 N-n+k+m) !(k-3 m) !(2 m) !}
\end{aligned}
$$

Известно [5], что

$$
|s(n, N)|=n ! \mathbf{P}\left\{v_{n}=N\right\},
$$

где $v_{n}$ - случайная величина, равная числу циклов в случайной подстановке степени $n$. Из теоремы 4.2.10 [3] и соотношений (16), (32) получаем, что

$$
\begin{aligned}
|s(n, N)| & =\frac{n !(-\ln (1-\lambda))^{N}}{N ! \lambda^{n} \sqrt{2 \pi(n-N)}}(1+o(1)) \\
& =\frac{n ! \exp \{n-N-5 \beta / 8-2 v / 9\}}{N ! \lambda^{n-N} \sqrt{2 \pi(n-N)}}(1+o(1)) .
\end{aligned}
$$

Используя формулу Стирлинга и равенства (16), (33), можно показать, что

$$
Z=\frac{\beta^{k}}{k !} e^{-\beta} e^{-v / 2}(1+o(1)) .
$$

Представим $S$ в виде суммы $S=S_{1}+S_{2}$, где суммирование по $m$ в $S_{1}$ и $S_{2}$ осушествляется в пределах $0 \leqslant m \leqslant A$ и $A<m \leqslant[k / 3]$, соответственно, а положительная постоянная $A$ будет выбрана позднее. Несложно показать, что

$$
S_{1}=\sum_{m=0}^{A}\left(\sqrt{\frac{27 k^{3}}{16 N}}\right)^{2 m} \frac{1}{(2 m) !}(1+o(1))=\operatorname{ch}\left(\sqrt{\frac{27 k^{3}}{16 N}}\right)+\delta,
$$

где величина $\delta$ может быть сделана сколь угодно малой выбором достаточно больших $A$, $n, N$. Легко видеть, что при выполнении условий теоремы

$$
27 k^{3} / 16 N=v^{2} / 4(1+o(1)) .
$$


Следовательно,

$$
S_{1}=\operatorname{ch}(v / 2)(1+o(1)) .
$$

Ясно, что сумму $S_{2}$ можно сделать сколь угодно малой, выбрав $A, n, N$ достаточно большими. Отсюда и из (31), (34), (35) следует первое утверждение теоремы 4. Второе утверждение доказывается аналогично с использованием (27)-(30), (33) при

$$
m_{2}=n-N-2 k-1, \quad m_{4}=2 m+1, \quad m \geqslant 0 .
$$

Доказательство теорем 5-7. Вероятность $\mathbf{P}\left\{\zeta_{N}=n, \xi_{i} \neq r, i=1, \ldots, N\right\}$ представим в виде

$$
\begin{aligned}
\mathbf{P}\left\{\zeta_{N}=n, \xi_{i} \neq r, i=1, \ldots, N\right\}= & \mathbf{P}\left\{\zeta_{N}=n\right\}-N p_{r} \mathbf{P}\left\{\zeta_{N-1}=n-r\right\} \\
& +\left(\begin{array}{c}
N \\
2
\end{array}\right) p_{r}^{2} \mathbf{P}\left\{\zeta_{N-2}=n-2 r\right\}-\ldots \\
& +N\left(-p_{r}\right)^{N-1} \mathbf{P}\left\{\xi_{1}=n-(N-1) r\right\}+\left(-p_{r}\right)^{N} \delta_{1},
\end{aligned}
$$

где $\delta_{1}=1$, если $n-N r=0$, и $\delta_{1}=0$, если $n-N r \neq 0$. Тогда из (2), (3) получаем, что

$$
\mathbf{P}\left\{\mu_{r}=0\right\}=1-N p_{r} \frac{\mathbf{P}\left\{\zeta_{N-1}=n-r\right\}}{\mathbf{P}\left\{\zeta_{N}=n\right\}}+R_{0},
$$

где

$$
R_{0}=\left(\begin{array}{l}
N \\
2
\end{array}\right) p_{r}^{2} \frac{\mathbf{P}\left\{\zeta_{N-2}=n-2 r\right\}}{\mathbf{P}\left\{\zeta_{N}=n\right\}}-\left(\begin{array}{c}
N \\
3
\end{array}\right) p_{r}^{3} \frac{\mathbf{P}\left\{\zeta_{N-3}=n-3 r\right\}}{\mathbf{P}\left\{\zeta_{N}=n\right\}}+\ldots+\frac{\left(-p_{r}\right)^{N} \delta_{1}}{\mathbf{P}\left\{\zeta_{N}=n\right\}},
$$

при этом, как несложно показать,

$$
0 \leqslant R_{0} \leqslant\left(\begin{array}{l}
N \\
2
\end{array}\right) p_{r}^{2} \frac{\mathbf{P}\left\{\zeta_{N-2}=n-2 r\right\}}{\mathbf{P}\left\{\zeta_{N}=n\right\}}
$$

Аналогично, из (2), (3) находим, что

$$
\mathbf{P}\left\{\mu_{r}=1\right\}=N p_{r} \frac{\mathbf{P}\left\{\zeta_{N-1}=n-r\right\}}{\mathbf{P}\left\{\zeta_{N}=n\right\}}-R_{1}
$$

где

$$
\begin{aligned}
& R_{1}=N p_{r}\left((N-1) p_{r} \frac{\mathbf{P}\left\{\zeta_{N-2}=n-2 r\right\}}{\mathbf{P}\left\{\zeta_{N}=n\right\}}\right. \\
&\left.-\left(\begin{array}{c}
N-1 \\
2
\end{array}\right) p_{r}^{2} \frac{\mathbf{P}\left\{\zeta_{N-3}=n-3 r\right\}}{\mathbf{P}\left\{\zeta_{N}=n\right\}}+\ldots+\frac{\left(-p_{r}\right)^{N-1} \delta_{1}}{\mathbf{P}\left\{\zeta_{N}=n\right\}}\right) \\
& 0 \leqslant R_{1} \leqslant 2\left(\begin{array}{c}
N \\
2
\end{array}\right) p_{r}^{2} \frac{\mathbf{P}\left\{\zeta_{N-2}=n-2 r\right\}}{\mathbf{P}\left\{\zeta_{N}=n\right\}} .
\end{aligned}
$$

Пусть выполнены условия теоремы 5. Если $N=2$ или $n-2 r<N-2$, тогда $R_{0}=R_{1}=0$. При $n-2 r \geqslant N-2$ и $n-2 r \geqslant \varepsilon n, \varepsilon>0$, используя (1) и лемму 4 , получаем, что

$$
\left(\begin{array}{l}
N \\
2
\end{array}\right) p_{r}^{2} \frac{\mathbf{P}\left\{\zeta_{N-2}=n-2 r\right\}}{\mathbf{P}\left\{\zeta_{N}=n\right\}}=o\left(N p_{r}\right)
$$


Если же $n-2 r \geqslant N-2$ и $n-2 r=o(n)$, то $r / n \rightarrow 1 / 2$,

$$
\begin{aligned}
\mathbf{P}\left\{\zeta_{N-2}=n-2 r\right\} & \leqslant(N-2) \sum_{l \geqslant(n-2 r) /(N-2)} \mathbf{P}\left\{\zeta_{N-3}=n-2 r-l\right\} p_{l} \\
& \leqslant \frac{N(N-2)}{(n-2 r) \ln n} \rightarrow 0
\end{aligned}
$$

и также справедливо соотношение (40). Утверждение теоремы 5 следует из (1), (36)-(40) и леммы 4.

Пусть теперь $m=n-r \geqslant N-1 \geqslant 2, m \rightarrow \infty, m=o(n)$. С помощью (1) и леммы 4 , получаем, что

$$
\begin{aligned}
N p_{r} \frac{\mathbf{P}\left\{\zeta_{N-1}=m\right\}}{\mathbf{P}\left\{\zeta_{N}=n\right\}} & =\frac{N p_{r}}{N p_{n}} \sum_{k_{1}+\ldots+k_{N-1}=m} \frac{\lambda^{m}}{k_{1} \ldots k_{N-1}(-\ln (1-\lambda))^{N-1}}(1+o(1)) \\
& =\frac{(N-1) !|s(m, N-1)|}{(\ln n)^{N-1} m !}(1+o(1)) .
\end{aligned}
$$

В [5] показано, что если $N=o(\ln m)$, то

$$
|s(m, N-1)|=\frac{(m-1) !}{(N-2) !}(\ln m+C)^{N-2}(1+o(1)),
$$

где $C$ - постоянная Эйлера, а при условии $m-\delta \sqrt{m} \leqslant N-1 \leqslant m, \delta \rightarrow 0$,

$$
|s(m, N-1)|=\left(\begin{array}{c}
m \\
N-1
\end{array}\right)\left(\frac{N-1}{2}\right)^{m-N+1}(1+o(1)) .
$$

Учитывая, что $R_{0}=R_{1}=0$, отсюда и из (36), (38), (41) получаем утверждения (1) и (6) теоремы 6, а утверждения (2)-(5) следуют из (32), (36), (38), (41) и теорем 4.2.2, 4.2.6, 4.2.8, 4.2.10 книги [3].

Если $N=2, m=n-r=o(n)$, то из (2), (3) находим,что

$$
\begin{aligned}
& \mathbf{P}\left\{\mu_{r}=0\right\}=1-\frac{2 p_{r} p_{m}}{\mathbf{P}\left\{\xi_{1}+\xi_{2}=n\right\}}, \\
& \mathbf{P}\left\{\mu_{r}=1\right\}=\frac{2 p_{r} p_{m}}{\mathbf{P}\left\{\xi_{1}+\xi_{2}=n\right\}} .
\end{aligned}
$$

Отсюда, из (1) и леммы 4 следует, в соответствии с замечанием 3, что утверждение (1) теоремы 6 остается справедливым, если $N=2, m=o(n)$.

Очевидно, что при выполнении условий теоремы $7 R_{0}=R_{1}=0$. Используя лемму 4 для оценки $\mathbf{P}\left\{\zeta_{N}=n\right\}$, а также (1), из (36), (38) получаем утверждение теоремы 7.

Для доказательства теоремы 8 заметим, что из определения обобщенной схемы размещения (см. (8.1.1) в [2]) и (2), (3) следуют равенства

$$
\begin{aligned}
& \mathbf{P}\left\{\mu_{n / 2}=0\right\}=\frac{\mathbf{P}\left\{\xi_{1}+\xi_{2}=n, \xi_{1}, \xi_{2} \neq n / 2\right\}}{\mathbf{P}\left\{\xi_{1}+\xi_{2}=n\right\}}=1-\frac{p_{n / 2}^{2}}{\mathbf{P}\left\{\xi_{1}+\xi_{2}=n\right\}}, \\
& \mathbf{P}\left\{\mu_{n / 2}=2\right\}=\mathbf{P}\left\{\xi_{1}=\xi_{2}=n / 2 \mid \xi_{1}+\xi_{2}=n\right\}=\frac{p_{n / 2}^{2}}{\mathbf{P}\left\{\xi_{1}+\xi_{2}=n\right\}}
\end{aligned}
$$

Отсюда, из (1) и леммы 4 получаем утверждение теоремы 8. 


\section{Список литературы}

1. Тимашёв А. Н., О распределении числа циклов заданной длины в классе подстановок с известным числом циклов. Дискретная математика (2001) 13, №4, 60-72.

2. Колчин В. Ф., Севастьянов Б. А, Чистяков В. П., Случайные размещения. Наука, Москва, 1976.

3. Колчин В. Ф., Случайные графы. Наука, Москва, 2000.

4. Павлов Ю. Л., Лосева Е. А., Предельные распределения максимального обьема дерева в случайном рекурсивном лесе. Дискретная математика (2002) 14, №1, 60-74.

5. Сачков В. Н., Комбинаторные методы дискретной математики. Наука, Москва, 1977.

Статья поступила 17.04.2003. 